\title{
Marchands moralistes (II)
}

\section{Ser Lapo Mazzei et Francesco Datini}

Le heurt des deux morales - morale chrétienne ascétique et mystique, morale marchande réaliste et pragmatique - est le ressort dramatique de la correspondance échangée de 1390 à 1412 par le notaire ser Lapo Mazzei et le marchand Francesco Datini ${ }^{469}$.

Qui sont les deux hommes en présence, à la fois opposés et liés pour l'éternité par un échange régulier de lettres : vingt ans d'une correspondance assidue, à laquelle la mort seule apporta un terme, plus de 400 missives ?

Tout d'abord le marchand, destinataire des lettres et objet de la correspondance, personnage plus passif qu'actif dans le drame. F. Melis, l'un de ses plus récents biographes, dit à juste titre que, de même que l'on juge l'artiste à ses œuvres, de même l'on doit juger le mercator à ses entreprises commerciales ${ }^{470}$. Or celles de Datini nous sont bien connues grâce à la survie de presque toutes ses archives, conservées à Prato : 1194 liasses ou registres, dont 574 livres comptables et 125000 lettres, soit plus de 600000 pages en tout! Le fair est exceptionnel. Les lettres, provenant de 267 localités différentes, procurent des renseignements sur 32 pays, dont la Libye, les Indes et la Chine. L'ensemble des documents recouvre la période 1363-1422, depuis la formation par Francesco de sa première compagnie, jusqu'à sa mort et au delà, pendant la période qui fut nécessaire pour régler son testament.

En somme, l'étude de cette masse énorme d'archives permet une solide reconstruction économique et historique du passé, durant un demi- 
siècle et pour une surface très étendue du globe. Elle permet on outre de bien connaittre le marchand Datini. Francesco est incontestablement un puissant mercator, qui dirige un ensemble de dix entreprises, dont sept commerciales (à Avignon, Pise, Florence, Gênes, Barcelone, Valence et Majorque), deux industrielles (tissage et teinture à Prato), une bancaire (à Florence) et une domestico-foncière (à Prato). Ces établissements juridiquement indépendants les uns des autres, ont en commun leur bailleur de fonds et leur patron (maggiore) en la personne de Datini. Celui-ci est donc le maître d'un complexe économique original, moins sensible à la conjoncture que la compagnie classique, plus souple, plus compétitif, plus propice à l'évasion fiscale : plus efficient en un mot. Depuis son cabinet, Datini contrôle et inspire la marche de ses entreprises, il donne par lettres ses ordres et ses directives, encourage ou réprimande ses collaborateurs, dont les tâches respectives sont étroitement délimitées et hiérarchisées et la soumission absolue.

Francesco n'a pas atteint d'emblée à cette puissance économique. A la différence de tous les marchands dont nous parlons, il n'a pas hérité du moindre fondaco. Il est un parvenu, dans le meilleur sens du terme. Il s'est formé lui-même. Le fait n'est pas commun dans une société aux structures déjà anciennes et très hiérarchisées. Fils d'un tavernier do Prato, Datini est orphelin en 1346, à l'âge de 13 ans. Au bout de deux ans d'apprentissage à Florence, il gagne Avignon. Durant trente deux ans, il demeure dans la cité des papes, où il fonde sa première compagnie, se perfectionnant dans son métier et élargissant peu à peu son horizon commercial. Après son retour dans la cité du florin, il crée progressivement le système d'entreprises décrit plus haut. Au cours des années, à force d'épreuves et de privations, il amasse une fortune énorme. La description de ses biens immeubles s'étend sur plus de 40 pages d'un libro mastro : à Prato, Francesco a 26 maisons, dans le contado il possède 36 pièces de terre, 7 bois et une villa. Ces biens immeubles, estimés à 12000 florins, ne représentent pas plus d'un cinquième de l'argent investi par Datini dans ses opérations commerciales.

C'est donc à bon droit que ses compatriotes l'appellent « le riche » ${ }^{471}$ et l'on comprend que certains de ses confrères le jalousent et le critiquent. L'un de ses correspondants ne lui écrit-il pas de Vérone : « Me trouvant avec des Florentins ici et en Lombardie et causant avec eux, comme il arrive, ils me disaient : - ton Francesco di Marco de Prato a les tripes bourrées d'argent " ${ }^{472}$. Au demeurant, Datini peut en Espagne rivaliser avec les Alberti et il est connu et respecté dans tous les milieux marchands de l'Occident. Un de ses facteurs lui écrit de Gênes : “ Il m'a été fait ici beaucoup d'honneurs par plusieurs compagnies floren- 
tines, et cela seulement grâce à l'estime que l'on vous porte. En vérité, vous avez ici une bonne et grande renommée de marchand " ${ }^{473}$. Consécration sociale de cette réussite : les Grands de ce monde, François Gonzague de Mantoue, Mathieu d'Humières, Leonardo Dandolo et Louis d'Anjou lui-même daignent s'arrêter dans sa riche demeure de Prato.

Cette évocation de Datini comme operatore economico est indispensable pour comprendre les données du drame qui l'oppose à Mazzei. Comme Francesco, ser Lapo est issu d'une modeste famille de Prato, mais les similitudes entre les deux hommes s'arrêtent là. Car Lapo par un choix délibéré de la médiocrité reste pauvre sa vie durant. Entré en 1370, à l'âge de 23 ans, dans l'étude d'un notaire de Prato, Mazzei, épouvanté par le caractère illicite des affaires qui y sont quotidiennement traitées ${ }^{474}$ et craignant pour son âme, quitte une situation pleine de promesses ${ }^{475}$. Il part alors pour Florence et entre un moment dans les bureaux de la Seigneurie. En juillet 1382, il est nommé coadjuteur de Coluccio Salutati, au salaire annuel de 40 florins d'or ${ }^{476}$. A plusieurs reprises, il est chargé de fonctions officielles : en 1383, il est notaire des prieurs, en 1391, il est secrétaire d'une ambassado envoyée à Gênes pour traiter avec Jean-Galéas. Ses collègues aussi consacrent sa valeur : de 1393 à 1410 , ils l'élisent plusieurs fois consul de leur corporation. Après avoir abandonné la chancellerie, ser Lapo entre au service de l'hôpital de Santa Maria Nuova, établissement d'assistance aux pauvres et aux malades, tout en demeurant le conseiller financier et administratif de quelques hommes d'affaires florentins parmi les plus grands : Niccolò da Uzzano, dont il rédige en 1399 le testament ${ }^{477}$, Guido del Palagio, un de ses amis intimes ${ }^{478}$, et Datini. Toutes ces fonctions ne lui procurent qu'une médiocre aisance, dont il se contente. Lorsqu'il meurt en 1412 , il ne laisse que quelques arpents de terre à ses enfants. Sa situation économique est donc sans commune mesure avec celle de Datini.

Notaire, Mazzei fait partie d'une corporation fort importante tant par son rôle économique que par sa culture. Selon Goro Dati, le notariat est une profession proprement florentine. Le chroniqueur ne dit-il pas avec quelque exagération qu'd Florence se trouve c la souche de tout l'art notarial exercé dans la Chrétienté et que de là proviennent ses grands maîtres, ses auteurs et ses écrivains " ${ }^{479}$. Et il ajoute que a la source des docteurs en droit est Bologne, cependant que celle des docteurs en notariat est Florence $\${ }^{480}$. Il apparaît donc que Florence s'est substituée à Bologne à la fin du $14^{\mathrm{e}}$ siècle pour la formation des 
notaires. Aussi bien ser Lapo écrit-il lui-même à un facteur de Datini : c Il y a des villes dans le monde où l'on se passe de tout mandat et de toute procuration, parce que les habitants n'y sont pas très experts en droit : ceci est véritable et j'en ai fait l'expérience à Gênes et à Venise, où il y a peu de juges et de notaires. Ailleurs, comme à Pérouse, Bologne, Florence et en d'autres cités, le mandataire ne pourrait remplir son office si les attestations de mandats ou de procurations n'étaient pas parfaitement fondées en droit $"{ }^{481}$. Dans la cité du florin, les notaires sont donc les collaborateurs indispensables des marchands, d'abord parce qu'aucun acte commercial n'a de valeur officielle s'il n'est pas enregistré sous leur seing, mais surtout parce que les mercatores font des notaires leurs conseillers administratifs, familiaux et même moraux. Aussi bien n'emploient-ils durant toute leur carrière que quelques-uns de ces hommes; tel Lapo di Giovanni Niccolini, qui en a trois seulement à son service, et successivement, entre 1378 et $1428{ }^{482}$.

Hommes de confiance par profession ${ }^{483}$, les notaires sont aussi hommes de culture. Ils connaissent la " grammaire », qui leur est indispensable pour étudier statuts et décrets administratifs et commerciaux ainsi que pour rédiger leurs actes. Ils possèdent un important bagage technique, fondé sur la connaissance approfondie de traités spécialisés, tels la Summa artis notarie, le Tractatus de notulis, le Flos testamentum ${ }^{484}$ de ser Rolandino Passeggeri (vers 1250) et les Commentaria ${ }^{485}$, la Practica iudiciorum ${ }^{486}$, et le Compendium ${ }^{487}$ de ser Piero Boattieri (1300 environ). Étudiant le droit romain pendant leurs années d'études, les notaires accèdent à la civilisation et à la culture latines. Ils sont en quelque sorte à mi-chemin entre les intellectuels et les marchands, participant aux préoccupations des uns et des autres. Ils comptent dans leurs rangs des hommes illustres comme Lapo Gianni, Brunetto Latini, Francesco da Barberino. Pour nous limiter aux notaires toscans contemporains de Mazzei, nombreux sont ceux qui contribuent directement au renouveau littéraire et philosophique comme correspondants assidus de Coluccio Salutati, leur maitre et leur confrère : ce sont, entre autres, ser Giovanni Cambini ${ }^{488}$, à qui Coluccio écrit en $13711^{489}$, possesseur d'un manuscrit des cuvres mineures de Sénèque, qu'il commente en des notes nombreuses et parfois pertinentes ${ }^{490}$, ser Castellano Castellani ${ }^{401}$, siennois, qui écrit à deux reprises en 1401 à Coluccio pour lui poser des questions littéraires ${ }^{492}$, ser Domenico di Salvestro ${ }^{493}$, qui compose pour sa corporation une épigramme destinée à être placée sous le portrait du chancelier de Florence, dans la salle d'honneur de l'Art des Notaires ${ }^{424}$, ser Antonio da Cortona ${ }^{495}$, ami de Benvenuto da Imola, qui lui confie la mission de présenter au même 
Salutati un exemplaire de son commentaire à la Divine Comédie ${ }^{496}$. Plus dignes encore d'être cités sont ser Piero Bonaccorsi ${ }^{407}$, fils d'un ami de Mazzei, qui compose un commentaire à la Divine Comédie, un Cammino di Dante ainsi qu'un traité fixant la date du voyage de Dante dans l'outre-tombe ${ }^{498}$, et ser Filippo Pieruzzi ${ }^{499}$, notaire des Riformagioni ${ }^{500}$, ami de Bruni, Manetti, Marsuppini et Traversari, dont Vespasiano da Bisticci nous dit qu'il connaissait parfaitement les arts libéraux, qu'il savait le grec et qu'il étudiait sans relâche les Écritures et les auteurs chrétiens ${ }^{501}$.

Lapo Mazzei est digne d'être compté au nombre des hommes que nous venons de citer. Il a droit à l'appellation d'homme cultivé. Ne multiplie-t-il pas tout au long de ses lettres des allusions aux auteurs classiques (Boèce ${ }^{502}$, Cicéron ${ }^{505}$, Sénèque ${ }^{504}$, Salluste ${ }^{505}$, Horace ${ }^{500}$, Tite-Live ${ }^{507}$, Valère-Maxime ${ }^{508}$ et Virgile ${ }^{509}$ ), aux auteurs chrétiens (saint Augustin ${ }^{510}$, saint Bernard ${ }^{511}$, saint François ${ }^{512}$, saint Thomas ${ }^{513}$ ), aux Écritures et aux auteurs en « vulgaire ) (Dante, Jacopone da Todi, Giovanni dalle Celle et les conteurs populaires ${ }^{514}$ ) ?

La culture de ser Lapo ne s'épuise pas en allusions. Dans sa correspondance, nombreuses sont les citations qui permettent de mesurer la profondeur et les limites de son savoir. Mazzei semble connaître les auteurs anciens surtout à travers des traductions, dont on sait qu'elles étaient fréquentes au Moyen Age : ainsi de Virgile, dont il cite des Morali, extraits de sententiae ${ }^{515}$. La culture classique de ser Lapo est done étendue, mais elle ne dépasse pas celle d'un c honnête homme " du Moyen Age. Cependant notre notaire fait parfois des citations très précises de Salluste ${ }^{516}$, Sénèque ${ }^{517}$ et même Virgile ${ }^{518}$. Il connaît en outre, de façon très approfondie, les auteurs religieux et cite à maintes reprises l'Ancien et le Nouveau Testament ${ }^{519}$. Enfin, il possède à fond la Divine Comédie, qui lui fournit matière à plus de treize citations 520 .

Mieux encore, nous apprenons par sa correspondance qu'il a une bibliothèque non négligeable et qu'il prête certains manuscrits et en emprunte d'autres. Sur les rayons de son cabinet voisinent le De Consolatione de Boèce ${ }^{621}$, les Épitres de saint Paul ${ }^{522}$, les Épîtres en a vulgaire ») de saint Jérôme ${ }^{523}$, les Évangiles ${ }^{524}$, les Laudes de Jacopone ${ }^{525}$, le Livre de saint Grégoire ${ }^{526}$, et la Vie de saint Thomas quand il alla servir le roi des Indes ${ }^{527}$.

Mazzei est en outre en relation avec un Bartolo, libraire ${ }^{528}$, et des copistes à qui il demande de reproduire certains manuscrits pour sa bibliothèque et celle de Datini ${ }^{529}$. Il fréquente même des professeurs de droit du Studio : messer Torello Torelli ${ }^{530}$ et messer Stefano di 
Giovanni Buonaccorsi ${ }^{531}$. Il a des rapports avec ser Coluccio, son ancien chef, à qui il fournit des anchois ${ }^{532}$ ou des épices et dont il révère profondément la science et l'éloquence ${ }^{583}$.

Enfin, la culture chrétienne de notre notaire, qui l'emporte en ampleur et en profondeur sur toutes ses autres connaissances, s'enrichit constamment grâce aux relations étroites qu'il entretient avec certains religieux, maîtres à penser du cercle des marchands mystiques florentins, dont Chiara Gambacorti, Giovanni Dominici, ami de Morelli 534, et surtout Giovanni dalle Celle ${ }^{535}$, dont nous verrons qu'il exerce une profonde influence sur la pensée et même sur le langage de notre notaire ${ }^{538}$.

Compte tenu de cette culture profonde et vécue, la correspondance que ser Lapo échange avec Datini ne peut que se distinguer, par le ton et les préoccupations, des autres correspondances marchandes.

Quant au langage d'abord, la différence éclate par comparaison avec deux lettres inédites de Giovanni et Bernardo Portinari, que nous avons choisies pour leur tonalité moralisante et philosophique, analogue à celle des missives de Mazzei. Le sermo cotidianus est le ton et le langage des deux mercatores. Aussi bien leurs lettres présentent-elles l'intérêt d'être d'excellents documents de cette prosa puntuale, dont nous avons déjà dit combien il est difficile de la caractériser pour le Trecento, tant les témoignages en sont rares, en dehors des livres comptables, lesquels sont souvent des traductions du latin et demeurent toujours très pauvres dans leur vocabulaire (technique) et leur syntaxe.

Le 25 août 1451, Giovanni Portinari envoie à son beau-frère une lettre de condoléances, à l'occasion de la mort d'un de ses fils :

“ Oे visto chome Ubertino, tuo fanciullo s'amalò alla Fonte, ma no dice di che male, e per modo che in pochi dì Domenedio se lo chiamò a ssé. Della qual chosa n'abiamo auti tutti e abiamo assai dolore e dispiacere, quanto di chosa ci potesse essere avenuto... Pure chonsiderando ch'a tutti ci chonviene andare a quel punto e, quanto più tempo ei presta, più pechati ne portiamo e più amaritudine portiamo in questo mondo misero, e ch'è suta volontà di Dio e a questo non è rimedio, vi priegho quanto so e posso siate chontento alla volontà di Dio e porti in pace. Ché per dispiacere e manichonia nollo riaremo, ma possiamo arogiere al danno e nostro Singniore lo abbia per male. Sicché piacciati essere chontento alla sua volontà o preghiamo Iddio ghuardi te e gli altri. E simile chonforta la Lisa per nostra [parte] e ch'ella sia chontenta a quello piace al nostro Signore, o lui priegho vi inchuori e choncedavi degli altri, poi s'è voluto quello a ssé, 
perché..........., e' truovasi a piè di Dio e libero delle pene di questo mondo misero. Or più non so che dirmi se no' che priegho Iddio vi dia pazienzja a voi e noi, e ch'assai passione ne portiamo. Xisto vi guardi ${ }^{537}$.

C'est une consolatoria du même style que l'on peut lire sous la plume de Bernardo Portinari, frère du précédent, à l'occasion de la mort de son propre père. Si dans la lettre de Giovanni les considérations demeurent exclusivement chrétiennes et familiales, par contre dans la missive de Bernardo les préoccupations marchandes réapparaissent bientôt. Le souci du profit l'emporte sur la douleur - pourtant réelle - car c'est un homme d'affaires qui est mort cette fois, et un vieillard, et non pas un enfant.

(Charo cogniato, a salvamento arivai qui, chome arai sentito ed [arai] auto la dolorosa nuova della perdita fatta del nostro charo padre, per la quale sono in gran passione, chome arai pensato. Né aspettavo tal nuova e troppo gran danno ne riceviamo nonostante l'età sua, ché le chose avean bisogno di lui ancora lugnamente. Ma poi ch'è suto piacere di Dio torcelo, ci chonviene rassegnarci alla volontà sua, e io chosì farò e attenderò a preghare per l'anima, la quale gli sia piaciuto ricevere nella [sua glo]ria, E volessi Iddio mi fossi ritrovato alla sua fine a chonfortare chotesta nostra vedova madre, la quale penso ne resta mal paziente, ma chonfido in voi e negl'altri nostri parenti e amici che l'avete chonsolata. $E$ io per lett. la chonforto, poi che per altro modo nollo posso fare. E voi priegho l'abiate per rachomandata [chome] sempre l'avete auto, o per lo simile Adoardo, al quale vi pregho tegniate le mani adosso a richordar[gli] quello vi pare sia di bisognio per chonservare el pocho ci à lasciato, ché lui mi schrive farà tutto [quello potrà] e chosì chredo farà, ché conoscierà ora meglio il bisognio suo e nostro. E quando vedesti facessi l'opoxito e che fosse bisognio io tornassi di chostà per salvamento dello stato nostro, vi priegho me n'avisiate, ché verrei subito e sanza lungho dire. Vi rachomando in tutta [choscientia], ché in voi chonfido più che in altri. E la Lisa chonfortate per mia parte : che voglia aver pazienzia alla volontà di Dio, non bisogna vi rachomandi ${ }^{538}$.

Les deux lettres sont intéressantes sur le plan du langage comme sur celui des idées. Bernardo et Giovanni écrivent comme ils parlent. Ils élident les a che » relatifs et déclaratifs, emploient la désinence en -i à la troisième personne du singulier du subjonctif imparfait, multiplient les ellipses et les anacoluthes, les liaisons par conjonction, etc. Ils négligent d'autres part les rapports logiques, les constructions rhétoriques, exprimant leurs idées au mépris de la correction grammaticale et de la clarté du discours. Ils n'ont pas médité leurs propos, n'ont 
pas composé de brouillon. Sur le papier, ils transcrivent les idées qui leur viennent à l'esprit, dans l'ordre et dans la forme où elles se présentent à eux : encore confuses, lourdes de sous-entendus et de ramifications mal exprimées. Chose étonnante de prime abord : les deux marchands ne s'expriment pas par parataxes, mais se lancent dans de longues phrases, dont il est parfois malaisé de discerner le commencement et la fin. D'où l'on voit mieux comment le style paratactique est chez Pitti un effet de l'art, le fruit d'une longue et consciente élaboration. Les idées exposées par les deux hommes d'affaires sont à l'image de leur " écriture » : restant au cas particulier - la mort d'un individu - sans souci de s'élever jusqu'à une véritable prise de conscience de l'universel, jusqu'au problème de la condition humaine. Les méditations philosophiques ne sont pas de leur propos. Les consolations elles-mêmes sont des lieux communs, usés par l'habitude, tant dans leur contenu que dans leur expression. En somme, les lettres de Bernardo et Giovanni Portinari demeurent des documents, intéressants et même émouvants, mais dépourvus d'ambition et de c dignité » littéraires.

Il n'en est absolument pas de même des lettres de Mazzei à Datini. Lorsqu'il écrit au marchand de Prato, le notaire songe à un public : il espère toucher son correspondant, mais aussi sa famille et le groupe de ses amis. Il écrit donc pour être lu, et lu à voix haute ${ }^{639}$. Il s'adresse en outre à ce cercle d'hommes d'affaires préoccupés de religion qui fréquente Dominici et Giovanni dalle Celle. Ce faisant, il ne peut employer la prosa puntuale des hommes d'affaires. Il se rapproche au contraire du style des prédicateurs. Son langage a de nombreux traits communs avec celui du moine de Vallombrosa et de Guido del Palagio tel qu'il apparaît dans des lettres échangées par les deux hommes à l'occasion du décès d'un fils de Guido.

Giovanni dalle Celle écrit au père meurtri : “ Voleati scrivere che tu non ti dolessi né maravigliassi, con ciò sia che la morte sia cosa naturale ad ogni vivente ; e ancora non posso. Voleati scrivere che noi entrammo nel mondo con questa condizione, che noi ne dovessimo uscire ; e che quello che noi riceviamo da Dio, rendere ci conviene ; e come questa vita ̀̀ una peregrinazione che, quando molto saremo iti, a casa ci conviene tornare, cioè a colui che ci mandò in questo mondo ; e non posso... ") ${ }^{540}$.

A quoi Guido del Palagio répond solennellement : « ... ricevetti vostra lettera, la quale più tosto e meglio si dee chiamare epistola : piena di tanta carità e di tanta affezione di cordiale amore, che scrivere non lo potrei con penna : piena di tanto dolore, quanto richiede l'afflizione di colui che ha perduto così benedetto unigenito figliuolo, come 
temporalmonte ho fatto io : poi piena di tanti conforti e di tanti buoni e santi ammaestramenti con ottimi consigli, che a me bastare dovrebbe, quella leggendo bene e seguitando la vostra dottrina, a sopportazione di tanti e tali dolori quanti sono quelli ne' quali al presente mi truovo ${ }^{541}$.

On mesure la différence qu'il y a entre ces propos et ceux des frères Portinari. Les parallèles, les figures de style se multiplient dans les lettres de Giovanni dalle Celle et de Guido dal Palagio, le vocabulaire y est typiquement ascético-mystique. Les deux (c épîtres ) (comme Guido a raison de les appeler) sont de véritables exercices religieux et elles sont dignes d'accéder aux anthologies littéraires. Or Mazzei fréquente assidûment les deux hommes; il lit leur correspondance et lui assure une certaine publicité : la tonalité et les préoccupations de ses lettres ressortissent à la même veine.

Le notaire florentin demeure pourtant à un niveau inférieur quant à l'c écriture $)$. On retrouve en effet dans sa correspondance certains traits typiques du sermo cotidianus : rhotacismes ( fragelli $^{542}$, sprendore ${ }^{543}$, obrigo ${ }^{544}$ ) sonorisation du -c- $\left(\right.$ regare ${ }^{545}$, galcina $\left.{ }^{546}\right)$, assimilation du -rdevant pronom enclitique dans les infinitifs oxytons (cancellallo ${ }^{547}$, ringraziallo ${ }^{548}$ ) et même une forme typique de Prato et de Pistoia (altre pour altri ${ }^{549}$ ). Mieux : ces formes populaires - peu nombreuses il est vrai - contaminent même le vocabulaire ascétique et mystique. Done, au moins par son langage, ser Lapo ne se distingue pas nettement de ses confrères marchands.

L'expression, par contre, est souvent digne de Giovanni dalle Celle, dont le style est très fidèle aux enseignements de l'ars dictaminis ${ }^{550}$. Ser Lapo a d'autant moins de peine à s'exprimer selon les règles do la rhétorique, que l'arte del dittare est l'une des matières enseignées aux notaires pendant leurs études. Les grands maîtres de l'art notarial, en effet, proposent à leurs étudiants des traités de rhétorique et des modèles de lettres. Ainsi ser Piero Boattieri, qui compose une Rosa novella ${ }^{551}$, un Compendium super vigenti sex varietatibus epistolarum ${ }^{552}$ et un recueil d'Epistole, formé d'épîtres historiques, de missives d'un notaire et de formules de lettres ${ }^{553}$. Bien plus, nombreux sont les collègues de Lapo qui ont composé des traités de rhétorique, depuis ser Antonio da Tempo ${ }^{554}$, auteur d'un $D_{6}$ rythmis vulgaribus ${ }^{555}$ jusqu'à ser Andrea Redusio da Quero ${ }^{556}$, auteur d'un manuscrit intitulé Grammaticae auctoribus rhetoricae atque notariae per annos VII, aujourd'hui perdu ${ }^{557}$. Mais Lapo lui-même ne propose-t-il pas à Datini des modèles de lettres à adresser aux citoyens influents de Florence pour obtenir lour appui politique et financier ${ }^{558}$ ? 
Ser Lapo est le plus souvent fidèle à l'enseignement qu'il reçut à Florence et Bologne. Sa culture lui procure les « flores » nécessaires à l'ornatus de son langage. Ainsi, par exemple, d'une lettre du 7 novembre 1395, dans laquelle il s'exclame : « Che è a dire che questo uomo sia stato nove anni per fare uno nidio... ! uomo c'ha auti tutti i diletti del corpo, le ricchezze e gli stati cognosce che sono nulla perché s'hanno a lasciare ! uomo ch'ha da Dio buona notizia di queste cose transitorie, con le parole sempre le sprezza e annulla! uomo di etade d'anni 65, come che si dia a creder di meno dieci, per ristorare l'opere vecchie, messo nel mezzo del verno a murare uno luogo che non arà mai fine, con tanta avidità, con tanta forza di volontà, con tanto affanno e ira e tribulazione, che non sapre' disegnallo con penna, ma tutto con la mente ogni dì veggo e intendo ! uomo che possiede tanto tesoro, che ne potrebbe fare uno fuoco e una acqua viva che saglirebbe insino a vita eterna ! uomo che trent'anni o più gli ha prestato Iddio tempo d'ordinare il modo del morire e istribuire il suo patrimonio, ed egli si troverà uno testamento col quale è reda il santo vescovo che fia di Pistoia ! ”569. On retrouve accumulés dans ces phrases plusieurs des ornamenti que recommande, entre autres, un fra Guidotto da Bologna ${ }^{560}$ : ornamento de gridare (exclamatio), ornamento de adomandare (interrogatio), articolo ${ }^{561}$, ridicimento (complexio), etc. Outre les ornamenti, les métaphores ${ }^{562}$, le vocabulaire ${ }^{563}$, les citations bibliques ${ }^{564}$, les latinismes ${ }^{565}$ et l'ironie sont significatives d'un effort délibéré accompli par Lapo pour être écrivain. Nous sommes ainsi bien loin du langage diffus des Portinari, de la parataxe chère à Pitti ou de la prose c boccaccesca » de Morelli. Lapo se veut l'émule des prédicateurs de Santa Maria Nuova ou de Santa Reparata, dont il fréquente assidûment les sermons et dont il admire l'éloquence ${ }^{566}$. Ne se définit-il pas luimême comme “ predicatore " 567 ?

Dans le passage que nous venons de citer, on ne relève qu'un trait typique du sermo cotidianus ${ }^{568}$. Peut-on dire que notre notaire écrivain, du fait de sa culture supérieure, s'exprime tout naturellement dans une langue plus châtiée que les marchands ? Non sans doute, et il faudra convenir que l'ornatus est chez lui aussi un effet de l'art, si l'on compare un fragment de lettre typiquement mercantile à l'épître déjà citée : “ Pensando del modo di certa procura ch'ha andare di fuori, mi venne in mente che diciate a ser Schiatta, ch'abbi in ricordo, che se non pone il nome del Papa, cioè il suo pontificato, quella che volete mandare a Vignone non sarebbe accettata, se chi avesse a piatir con voi là, o avesse a pagare, volesse opporre al mandato; o e' sarebbe a provare là che qua non s'usa. Penso bene è da tanto, che non vi 
mancherà nulla. E ricordar qui papa Chimenti etc., non so come s'è onesto, a chi 'l mettesse in carta $"{ }^{569}$. Lorsqu'il traite d'affaires, ser Lapo redescend instinctivement au niveau d'une prosa puntuale dont il est inutile de noter une fois encore les traits caractéristiques. Il faut cependant relever le ( etc » de la dernière phrase, car cette locution, typique de Pitti, est très rare chez Mazzei, qui ne se refuse pas d'ordinaire à l'élan rhétorique et littéraire. Ici le « etc » réapparaît parce que le notaire ne se soucie plus que d'être rapidement et utilement compris.

Davantage : certaines lettres montrent que l'écriture diffère selon les circonstances et les préoccupations. Dans un billet du 17 décembre 1400 par lequel Mazzei conseille à son lecteur d'abandonner les poursuites contre un mauvais payeur, on distingue deux tonalités différentes qui se succèdent brutalement. Le sermo cotidianus est le langage des considérations mercantiles et des raisons techniques. Le ton s'élève et le rythme devient plus lent lorsque le notaire aborde des arguments moraux. L'exclamation ma de est le point d'articulation qui permet le brusque changement de registre : “ Torno a Tanfuro. $E$ dicovi non si può far in modo alla Mercatanzia che e' non esca sodando bene. Ma se aveste chiarito ch' $e$ ' v'ha a dare, e avestene sentenzia, allora si può fare al Podestà di qui pigliare il debitore ; e non può mai uscire se non paga. Ma de ! non entrate or più adentro : lasciate star così ; ella sta bene : pensate a vivere nella memoria di Dio più che potete $!$ ' ${ }^{570}$.

Lapo a done bien droit à ce titre de prédicateur auquel il prétend. De l'art oratoire il emploie toutes les ressources et les figures. Et il est très rare que, conformément à certaines de ses promesses, il réussisse à “ mettre des rênes à sa langue $)^{571}$. Au contraire, il multiplie les antithèses, les images et les métaphores, les comparaisons et les parallèles. La plupart de ses lettres s’élèvent et se maintiennent consciemment sur le plan de la littérature. La seconde missive qu'il adresse à Datini, se hausse déjà à ce style qui demeure constant dans sa correspondance avec le marchand de Prato. Autour de l'antithèse centrale, nettement posée, s'organise un système d'images et de métaphores : “ Sogliono gli amici e' mercatanti delle cose del mondo fare somma letizia quando si fa alcuno grande acquisto di queste cose che ci sono date, spesse volte, a brieve tempo. Io, per grazia di Dio, lo fo nella mente e nel cuore quando sento raunare tesoro eterno ${ }^{3571}$ bis. Le ton et le langage ne changent pas à vingt ans de distance, dans l'une des ultimes missives de la correspondance Mazzei-Datini. Seule l'accumulation - presque baroque - des métaphores dans la dernière distingue les deux lettres : (c) Il vostro stomaco non sente sapore delle verità scritte di Dio, e isti- 
mate che la grazia, come rugiada di cielo, caggia in bocca, come una pera, tenendo forte $i$ denti $){ }^{572}$.

Lapo est donc un habile rhétoricien. L'une de ses plus ferventes protestations d'amitié adressées à Datini se développe sur le modèle des formulaires proposés par Guido Faba et ses confrères. Ne peut-on en effet rapprocher cette exclamation de Mazzei du De amico ad amicum qui repetit acomodari, responsivum composé par le (dettatore " bolonais ${ }^{573}$. “ In ciò ho auto fidanza con voi, per quanto m'avete donata la vostra amistà : che, come l'ho cara, Iddio il sa ; e 'l cuore mio il tace, ché non pensa saperlo mostrare 》s ${ }^{574}$.

Un rapide inventaire des figures qui se multiplient dans la correspondance de Mazzei peut fournir matière à déterminer les tonalités de son langage et à décrire son univers mental et littéraire. Les images qu'il développe embrassent le ciel et la terre. Ses perspectives mettent en question l'univers tout entier. Évoquant la vie terrestre, Lapo emploie des expressions comme “ pièges » ${ }^{575}$, “ vents et tempêtes » ${ }^{576}$, “ bois sans fond ${ }^{577}$, « fureur ${ }^{578}$ et « mer » ${ }^{579}$. Représentant la société humaine, il parle de (c vilenies » ${ }^{580}$, ( folies » ${ }^{581}$, ( mélancolies » ${ }^{582}$, “ félonies » ${ }^{583}$, “ hontes ${ }^{584}$, etc. Lorsqu'il fait allusion à la mort, son souci constant, il la considère comme instant de vérité, porte franchie sans retour ${ }^{585}$ après le bref (c pélerinage » ${ }^{586}$ terrestre, s'ouvrant sur une éternité conçue comme (c trésor 》 ${ }^{587}$, “ allégresse » ${ }^{588}$, (c floraison 》 ${ }^{589}$, ( paix 》 ${ }^{500}$, (c vérité ) ${ }^{591}$, ( parfums ) ${ }^{592}$ et (c amour ) ${ }^{593}$.

L'univers de Lapo est, on le voit, tout chrétien, ascétique et mysstique. Tirées d'un registre au fond peu étendu, les images qu'il emploie sont traditionnelles ; cependant, dans leur évidence même, dans leur répétition et surtout dans leur conviction (elles ne sont pas gratuites, mais profondément senties), elles retrouvent vie et vigueur. L'élan de la foi les ressuscite en quelque sorte, comme chez les écrivains mystiques contemporains, Dominici, Francesco d'Albizzo, Leonardo Giustinian ${ }^{584}$. Ainsi de cette lettre, laquelle définit avec un lyrisme indéniable le premier degré de l'ascension mystique, qui est viduité des sens et de l'esprit, obtenue dans la solitude : c Padre, oggi e solo in terra, quello vostro delle pecore, come figliuolo, essendo in sommo silenzio, nella pace della notte, raccomandandovisi, vi saluta, con desiderio né basso né vile, di vedervi nell'amor di Dio che v'ha creato, e andare per le vie che non va egli, né gli altri amadori del mondo ${ }^{595}$.

Tout pleins de Dieu qu'ils soient et de mépris du monde, les esprits mystiques demandent au moins à la terre de leur fournir des images, les plus fortes et les plus terribles. Certes, Lapo n'est pas Jacopone. Cependant il retrouve, à un niveau plus modeste, lo « réalisme » des 
grands mystiques et cela justement à partir du sermo cotidianus des marchands qu'il fréquente. Cette c redécouverte » n'est due ni au hasard, ni à l'instinct, mais à un choix conscient et délibéré : elle répond à la volonté qui anime Mazzei de toucher ses auditeurs grâce à des expressions volontairement triviales. A la fin d'une longue admonestation, par exemple, Lapo se ressaisit et fait un énergique effort d'humilité : (c Vi priego e conforto, come che vile e fracidoso sia, e presuntuoso a scrivere di tal materia, che è come se un porco o una capra volesse parlare del tessere la seta ${ }^{506}$. De la même façon, le mépris et l'horreur que Lapo éprouve pour le corps et la chair lui arrachent des exclamations brutales, sinon très originales : “ Pensa che vivere sia empiere, e votare, e scoppiare " ${ }^{597}$, (c i bevitori ghiottoni, de' quali il ventre è Iddio ${ }^{598}$.

Telle qu'elle apparaît déjà à travers ces exemples, l'inspiration ascétique et mystique n'est pas très originale chez Mazzei. Lapo fonde son ascétisme sur le dualisme implicite des versets fameux de Luc et de Matthieu : c on ne peut, écrit-il, servir deux maîtres à la fois, c'est-àdire Dieu et le monde, mais, traiter Dieu comme maître et le monde comme serviteur, cela on peut et on doit le faire $"{ }^{599}$. Le choix ainsi fait par Lapo de façon nette et défnitive est rappelé à toute occasion. Il implique le mépris du corps et s'accompagne du plus grand dédain pour le monde et les hommes : “ Voyez à combien de choses nous pensons pour satisfaire notre misérable corps qui, à bien y réfléchir, pue déjà » ${ }^{600}$. Lapo s'exclame ailleurs : "Sachez bien que nous sommes par nature poussés depuis notre jeunesse à être méchants, à nous préoccuper des richesses, de la chair et de la terre ${ }^{601}{ }^{60}$. En somme, notre homme considère de façon classique que la vie terrestre n'est qu'un passage, lequel n'a de sens qu'en fonction de la vie future. Il ne craint pas la mort, pas plus pour lui-même que pour ses enfants et ses amis : “ Ni pour mes amis, ni pour ma femme, ni pour mes enfants, mère ou frère, je ne suis décidé à me désespérer, mais à remercier Dieu de tout " ${ }^{602}$. Ainsi, durant l'épidémie de peste de 1400, qui vide la cité de ses habitants et en premier lieu de ses hommes d'affaires (Datini est des premiers à s'enfuir), Mazzei attend sereinement la mort : « Ici il meurt beaucoup de monde... Que Dieu nous aide à bien mourir, par sa miséricorde $){ }^{603}$.

Ce sont là les accents d'un ascétisme profondément senti. Bien qu'il vive dans le monde, Mazzei est un véritable ascète. Mais, à en croire sa correspondance avec Datini, il n'atteint que rarement aux degrés supérieurs de l'élan mystique. Cependant il s'abandonne lui-même et abandonne les siens à la Providence. Il se met en toute confiance entre 
les mains de Dieu : “ J'ai trois ou quatre florins sur quarante setiers de terre, avec onze enfants... Je ne crois pas qu'il y ait au monde un homme qui sache moins se plaindre que moi. Si je mourais, ils iraient mendier leur pain... Mais Dieu passe d'abord ; qui dit dans l'Évangile qu'il se soucie des moineaux, et d'autant plus de donner aux hommes leur pâture $)^{604}$. Cette deditio à la volonté divine dépasse le renoncement ascétique aux hommes et à la terre : elle est l'un des premiers degrés de l'échelle mystique. Aussi bien la peste, cette pierre de touche, fournitelle à Mazzei l'occasion d'un abandon plus parfait à la volonté de Dieu, plus parfait parce que dramatique, décidé à la suite d'un cruel débat : c Pensez, écrit-il à Datini réfugié à Bologne, combien mon cœur so fendait à voir les petits en larmes et leur mère en mauvaise santé, et à entendre les paroles de l'aîné. Et songer à trois morts ! Mais, comme je les vis si bas qu'ils ne pouvaient en réchapper, j'en pris mon parti et remerciai Dieu de la grâce qu'il me faisait... Je suis plus heureux que s'il leur était arrivé autre chose. Maintenant qu'ils ont quitté cet océan de douleurs et qu'ils se trouvent sur la rive de vérité, j'en loue Dieu ${ }^{605}$.

Cependant jamais ser Lapo ne semble atteindre au ravissement mystique. Les visites de Dieu auxquelles il fait une fois allusion ne sont rien de plus que des épreuves ascétiques : « Dieu, dont je vois par un signe qu'il ne m'a pas abandonné, mais qu'il me visite souvent, car pas plus tard qu'avant hier il voulut un autre de mes enfants $\gg{ }^{606}$. En somme, si l'élan ascético-mystique du notaire a sa noblesse, d'autant plus grande que l'homme est resté mêlé au monde de par sa profession et ses conditions mêmes d'existence, il n'est pour cela ni neuf ni profond. $\mathrm{Ou}$, pour parler plus exactement, l'originalité de ce mysticisme ne doit pas être recherchée dans son essence mais dans ses modalités.

Dans ses élans de foi, Mazzei garde, en effet, des accents humains qui sont typiques de la religiosité des temps nouveaux selon les analyses de D. Coppola ${ }^{607}$. Sa foi n'a pas les accents de la passion mystique, mais le ton plus modéré, plus " terrestre ", d'un Feo Belcari ou d'un Leonardo Giustinian. Elle n'est ni folie, ni certitude dogmatique, mais se développe sur le plan de la morale en acte.

Car la religiosité de Lapo est sans prétentions, mesurée : son ascétisme et son mysticisme sont éprouvés aux critères du juste milieu et du raisonnable, typiques de l'humanisme marchand. Et ils se fondent non pas sur une vision abstraite, a priori, du monde et de ses turpitudes, mais sur une expérience vécue et profonde de l'univers des hommes.

Tout mystique est avide de convaincre. Lapo n'échappe pas à la règle. Mais il ne cherche pas à envoyer ses correspondants au cloitre ; il 
n'espère pas leur faire revêtir la bure, se contentant de les ramener sur une voie plus chrétienne : “ Le sage, écrit-il, doit savoir se vaincre et ne pas se laisser aller à ses désirs, mais faire les choses avec mesure et tempérance... » ${ }^{608}$. Et il dit ailleurs : “ Je ne vous dis pas de vous faire frère ou prêtre ; mais je vous dis de modérer votre vie " ${ }^{609}$.

Ser Lapo est d'autant plus modeste dans ses ambitions qu'il se veut ( convertisseur ) d'âmes mercantiles. Telle est la mission qu'il s'attribue en ce monde. De son aveu même, il est très bien placé pour assumer cette tâche : “ J'ai vu venir... à mon bureau plusieurs marchands qui chaque année font l'aumône, compte tenu de leur bilan, d'une bonne partie de leurs gains. Et, pour faire une bonne aumône, ils ont demandé mes conseils sur ce que j'en sais " ${ }^{610}$. Ainsi Mazzei se préoccupe du salut d'un grand nombre de mercatores florentins. Certains viennent de leur propre initiative le consulter; à certains autres il impose ses conseils et ses directives spirituelles : c'est le cas de certains facteurs de Datini, tel Luca del Sera ${ }^{611}$, Cristofano da Barberino ${ }^{612}$, Simone d'Andrea ${ }^{\text {B13. }}$.

Datini est d'abord un disciple parmi les autres de notre pêcheur d'âmes. Mais il devient peu à peu le premier de ses tourments. L'arracher aux préoccupations mercantiles, le défendre des emprises du monde, tel est lo motif essentiel des épîtres que Lapo lui adresse. La correspondance entre les deux hommes devient un contrasto entre la morale chrétienne du premier et la praxis marchande du second.

Lapo connaît parfaitement les données du drame et les dispositions de son adversaire. Il lui accorde volontiers la forte personnalité que tous lui reconnaissent, discernant en lui des qualités exceptionnelles d'intelligence et de volonté : « Je vous ai toujours trouvé une grande intelligence et un jugement subtil $"{ }^{614}$. Il ne lui refuse pas non plus un penchant naturel pour le bien, mais note qu'il ne s'exprime qu'en paroles et n'atteint jamais à une parfaite adhésion à la vie chrétienne.

Davantage, Lapo discerne clairement la cause des velléités de Francesco et il va répétant qu'il est trop marchand pour être bon chrétien : " Si vous aviez à ne vous occuper que de laines... je crois que vous mourriez marchand " ${ }^{615}$. Bonnes intentions et heurenses dispositions ne peuvent arriver à réalisation par la fante des affaires, qui jamais ne laissent libres l'esprit et le cœur du mercator. Lapo lui reproche sans cesse ses multiples occupations, les centaines de lettres qu'il reçoit chaque jour des confins du monde. Il le condamne pour sa dureté en affaires et son âpreté excessive au gain. Selon ses dires, Datini vit dans la crainte d'être trompé et volé et il exige d'avoir son compte, même s'il est moins empressé à payer son dû. “ Vous êtes si avide, lui 
écrit-il, et vous désirez tant que pas un denier ne se perde, vous craignez tant qu'une seule brique ne soit placée de travers... que l'on ne déplace pas une civière sans que vous y touchiez, criant et vous tourmentant $)^{616}$. Au demeurant, Mazzei voit bien que les occupations mondaines - trafics, procès, soucis d'impôts, banquets, constructions de palais, achats de terres et de maisons - ne laissent guère à son correspondant le temps de penser à son âme. Aussi déclare-t-il qu'il est aussi difficile de redresser le caractère de Francesco et de corriger ses mauvaises dispositions que de courber dans le sens contraire une poutre incurvée durant plus de cent ans par une lourde charge ${ }^{617}$.

Pourtant notre avocat de Dieu ne renonce pas un instant à son entreprise. Afin d'atteindre son but, il utilise un nombre réduit d'arguments qu'il « martelle ) sans relâche, selon sa propre expression, dans l'espoir de les faire pénétrer dans la tête de son interlocuteur. Ainsi il lui rappelle que les richesses sont inutiles, passagères et fugaces, comme les plaisirs et les honneurs qu'elles procurent. Il affirme encore que les félicités de l'âme sont bien plus profondes et plus durables que les satisfactions du corps.

Pour son bon combat, Mazzei cherche et trouve des alliés. Il requiert l'appui de Margherita, l'épouse de Datini. Dès 1386, c'est-à-dire avant d'avoir rencontré le notaire, Margherita, parfaitement consciente du drame posé par le salut de son époux, lui écrit : “ Je voudrais que tu ne sois pas toujours ce que tu as été depuis que je te connais, un homme qui n'a jamais cessé de se tourmenter l'âme et le corps. Tu dis, tu répètes sans cesse, que tu vas embrasser une vie de bien dans le mois ou dans la semaine à venir. Tu dis cela depuis dix ans et tu me parais moins prêt que jamais à te reposer ${ }^{818}$.

De saintes personnes viennent, de leur propre initiative, au secours du notaire et de Margherita pour conspirer au salut du mercator. Elles appartiennent au groupe des conseillers des marchands mystiques dont nous avons parlé plus haut. Ainsi Giovanni dalle Celle envoie plusieurs lettres à Datini pour lui rappeler que le salut s'obtient par les cuvres, qu'il n'est pas octroyé et que les hommes d'affaires sont particulièrement exposés aux entreprises du démon ${ }^{619}$. Le ton et le langage sont ceux qu'emploie Manzei. Pareillement, Chiara Gambacorti pousse Datini à songer davantage à son âme et moins à ses trafics : " Aidezvous vous même, lui écrit-elle, et ne vous privez pas d'un tel bonheur à cause de vos grandes préoccupations d'affaires " ${ }^{620}$.

Aussi bien le cas Datini peut-il passer pour désespéré. Notre mercator ne voit pas où sont ses fautes. Il croit fermement qu'il se comporte en bon chrétien dans la mesure où il fait régulièrement l'aumône et 
accomplit ses obligations religieuses. Il est persuadé qu'on le comprend mal. Un véritable dialogue de sourds s'engage entre lui et ses conseillers mystiques. Lapo peut à juste titre prétendre que son ami a les oreilles bouchées ${ }^{621}$. Les arguments avancés par le mercator - impossibilité d'abandonner brutalement ses entreprises, efforts accomplis pour aider les pauvres, joie (ascétique !) qu'il éprouve à être persécuté par son percepteur ${ }^{622}$ - ne peuvent pas toucher le notaire, parce qu'ils ne correspondent pas à ses préoccupations et ne répondent pas vraiment à ses exhortations. De la même façon, les raisons de Mazzei ne satisfont pas Datini, car elles ne sont pas une réponse à ses problèmes d'homme d'affaires. En outre, Datini est d'autant plus attaché à la terre et à ses joies qu'il est un parvenu. Qu'il se soit fait lui-même, c'est ce qui exaspère ses défauts comme ses qualités mercantiles. Il ne peut avoir le détachement de certains Alberti par exemple ${ }^{623}$.

Au fond, Mazzei et Datini ne peuvent s'entendre. Si la foi chrétienne apporte à ser Lapo la solution à presque tous ses problèmes - sauf les problèmes commerciaux ${ }^{624}$ - elle n'est plus pour Francesco qu'une suprastructure. Les impératifs de la morale économique chrétienne en particulier (juste prix, usure ${ }^{625}$ ), ne résolvent pas ses problèmes. Plus ou moins consciemment il leur oppose la nouvelle éthique marchande : de réussite terrestre d'une classe et de certains individus, telle que nous l'avons définie ${ }^{628}$; éthique dans laquelle đle bien acquis » est oublié en faveur du profit, du bénéfice à tout prix.

Cependant Mazzei semble marquer peu à peu des points sur son adversaire, au moins dans le domaine de ses dispositions testamentaires. Car, Datini n'ayant ni enfants ni descendants, le but de Lapo est de destiner à des fins chrétiennes des richesses très paỉennes. Dès sa première rencontre avec Francesco, il veut le convaincre de créer une institution d'assistance aux pauvres et aux malades. Au fil des années il relève des progrès dans l'état d'esprit de son correspondant. En 1398, il n'en est encore qu'à lui conseiller de mettre quelques mots en ce sens sur son testament ${ }^{627}$. L'année suivante, il note que Francesco commence à laisser aller le monde ${ }^{628}$. En 1409 , il se réjouit à la pensée d'une évolution peut-être définitive chez son interlocuteur : « Vous sortez, lui écrit-il, de la fièvre et de la pauvreté, où sans doute vous vous trouviez, et vous devenez riche de la grâce de Dieu, d'une conscience et de biens indestructibles : et vous passerez paisiblement par la forêt du monde, si pleine d'embûches " ${ }^{629}$. Cependant, ce n'est qu'en 1410 que, d'un homme malade, Mazzei obtient le testament fondant l'hospice de Prato. Peut-on parler de victoire du mystique sur l'homme d'affaires ? Sans doute faut-il juger qu'il s'agit d'un triomphe 
de la mort proche plus que de la foi : en tout cas, d'une « elemosina del capezzale ${ }^{630}$, à quoi Lapo accordait explicitement peu de valeur. Non pas tant, donc, conversion que concession, laquelle impressionne pourtant les collaborateurs de l'homme d'affaires, qui n'ont probablement jamais cru vraiment à son complet revirement ${ }^{631}$.

Sur cette demi-victoire s'achève la correspondance Mazzei-Datini, heurt de deux dialectiques discordantes : mercantile et chrétienne. Par son fond comme par sa forme, le contrasto est intéressant, éclairant. Surtout, il fournit à ser Lapo l'occasion de composer une oouvre qui n'est pas négligeable : non seulement « testo di lingua », comme le veut son éditeur, mais aussi témoignage d'une vocation et d'un talent d'écrivain. Ser Lapo se sait différent de la plupart de ses confrères ${ }^{632}$. Il n'a pas tort. Il doit être compté, à un niveau plus humble sans doute, dans la lignée des grands notaires écrivains qui va de Brunetto Latini jusqu'à Coluccio Salutati en passant par Lapo Gianni. 